\title{
IMAGE-BASED BAS-RELIEF GENERATION WITH GRADIENT OPERATION
}

\author{
Meili Wang, Jian Chang, Junjun Pan,Jian J. Zhang
}

The National Centre for Computer Animation Bournemouth Media School

$$
\begin{aligned}
& \text { Bournemouth University } \\
& \text { Talbot Campus, } \\
& \text { Poole, Dorset BH12 5BB } \\
& \text { United Kingdom }
\end{aligned}
$$


|Technical Report TR-NCCA-2009-XX

ISBN : $1-85899-123-4$

Title: image-based bas-relief generation with gradient operation

Authors: Meili Wang, Jian Chang, Junjun Pan, Jian J. Zhang

Key words and Phrases: Bas-relief, Gradient operation, Unsharp masking, Poisson equation

Abstract:

Bas-relief is a type of sculpture artwork which is carved into a plane or a surface,

traditionally created by hand. Recently, computers have been adopted to assist the design

and production of relief works. In this paper, we propose a novel method to generate the 3D mesh of a relief based on a 2D image input. Gradient operations, such as magnitude

attenuation and unsharp masking, are introduced to convert an image into a relief. The

Poisson equation is solved for the construction of the depth information of a relief from

the tuned data. The final output as geometric mesh enables artists or designers to add

|additional texture and material features for their relief design. More importantly, such a

mesh can be directly used for computer aided manufacturing as input.

Report date: 01 March, 2009

Web site to download from: http://eprints.bournemouth.ac.uk/

The authors' e-mail addresses: mwang@bournemouth.ac.uk

Supplementary Notes: <Accepted by The International Association of Science and Technology

for Development (CGIM 2010)>

The National Centre for Computer Animation

Bournemouth Media School

Bournemouth University

Talbot Campus,

Poole, Dorset BH12 5BB

United Kingdom 